\title{
IL COMPLESSO MONASTICO DI S. MARIA D'AURONA. ARCHITETTURA E LITURGIA A MILANO TRA ETÀ LONGOBARDA E CAROLINGIA
}

\section{ROBERTO CASSANELLI}

UDC: $73.033 \cdot 4(450)$ 726.71/450.25)"07"

Original scientific paper

Manuscript received: 31. 10. 2016.

Revised manuscript accepted: 17. 01. 2017.

DOI: 10.1484/J.HAM.5.113710
R. Casanelli

Università Cattolica del Sacro Cuore Largo A. Gemelli, 1 20123 Milano, Italia robertocassanelli@virgilio.it

During the building works carried out between 1868 and 1869 for the construction of the head-offices of the Cassa di Risparmio delle Provincie Lombarde in Milan, a large mixed group of Early medieval and Romanesque sculpture fragments was uncovered (now in the Sforza Castle Museum). Thanks to topographic coincidences, this group was immediately connected to the so called Aurona nunnery, a monastery probably founded by the Lombard king Liutprandus in the first half of VIII century, that took its name from the unfortunate king's sister, Aurona, mentioned by Paul Deacon in his Historia Langobardorum. However only in 1944 Alberto de Capitani d'Arzago found the plan of the old building, giving finally an architectural frame to the fragments. Many doubts still remain on the chronological distribution of the pieces, their arrangement in the building and their liturgical function. Nevertheless, some new speculations can be now proposed after the cataloguing of all pieces in the museum's stores.

Keywords: Early medieval nunnery, Early medieval architecture, Lombard sculpture, Lombard Kingdom, Milan.

Nel corso degli scavi per le fondamenta del palazzo della Cassa di Risparmio delle Provincie Lombarde, che tra 1868 e 1869 si stava costruendo a Milano su progetto dell'architetto Giuseppe Balzaretti nella vasta area occupata dal palazzo del Genio militare lungo l'attuale via Monte di Pietà (già Contrada dei Tre Monasteri, fig. 1$)^{1}$, riemerse una vasta congerie di materiali lapidei ascrivibili a un ampio arco di tempo dall'età romana al Rinascimento. Già in precedenza peraltro sono registrati rinvenimenti. Nel maggio $1868 \mathrm{i}$ fratelli Tensi, proprietari di un giardino adiacente all'area, donarono al Museo Patrio di Archeologia «sei frammenti di scultura architettonica di stile lombardo»² e un gruppo

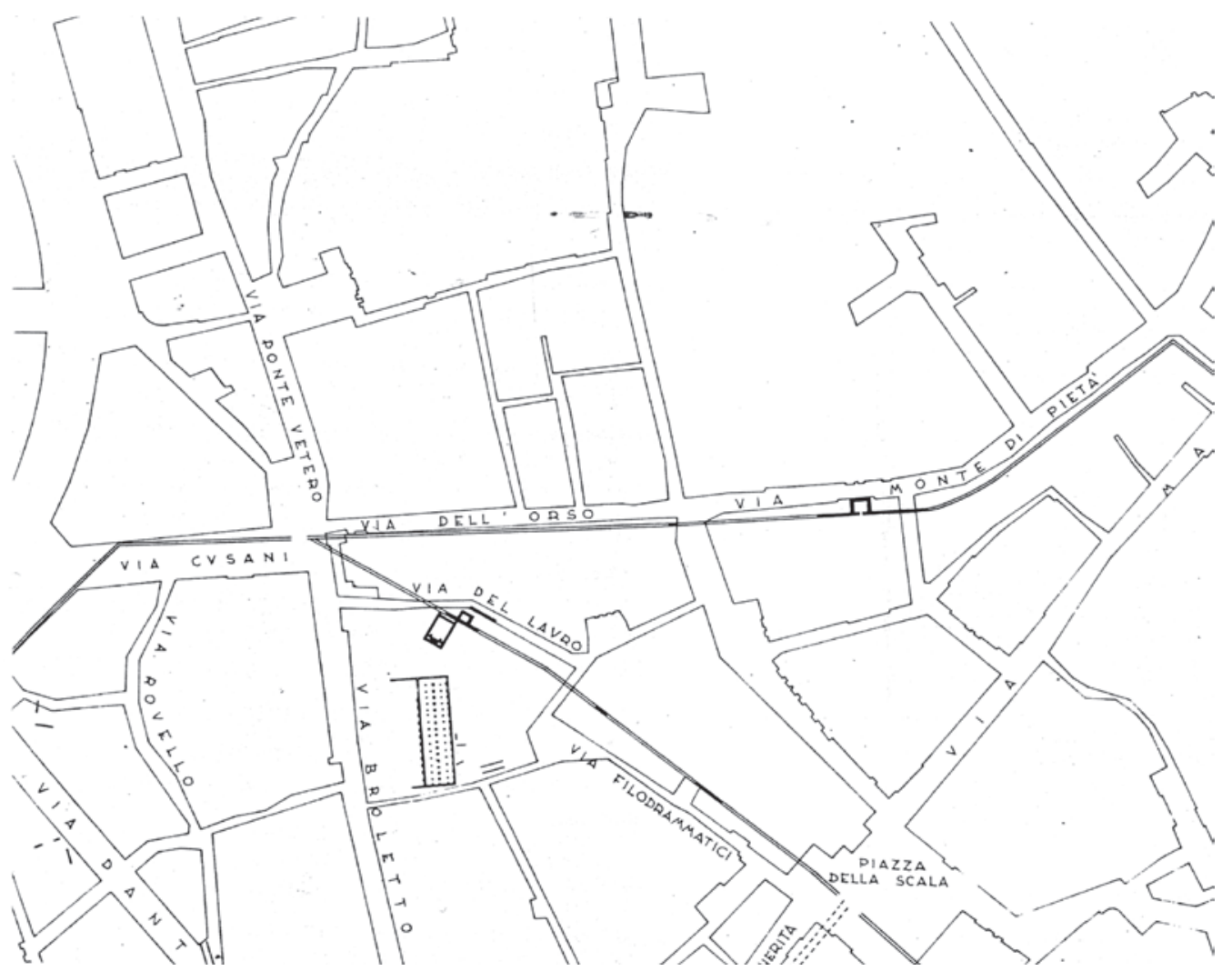

Fig. 1. Percorso della cinta muraria romana di Milano nel punto d'innesto con l'addizione tardoantica (dalla Forma Urbis Mediolani).

${ }^{1}$ Il palazzo è documentato da una fotografia di Luigi Sacchi anteriore al 1861, riprodotta in G. LANDRIANI, Gli avanzi della basilica di S. Maria in Aurona a Milano. Rilievi e note del prof. Gaetano Landriani, testo dell'arch. Luca Beltrami con prefazione del prof. Fernand de Dartein, Milano, 1902, rist. anast. in R. Cassanelli, M. David (a cura di), Archeologia e medioevo a Milano. Gaetano Landriani e la scoperta di S. Maria d'Aurona, Cinisello B., 2000.

${ }^{2}$ Milano, Civiche Raccolte d'Arte, Archivio. Catalogo Museo patrio archeologico, II, nn. 1619-12 e ss. 


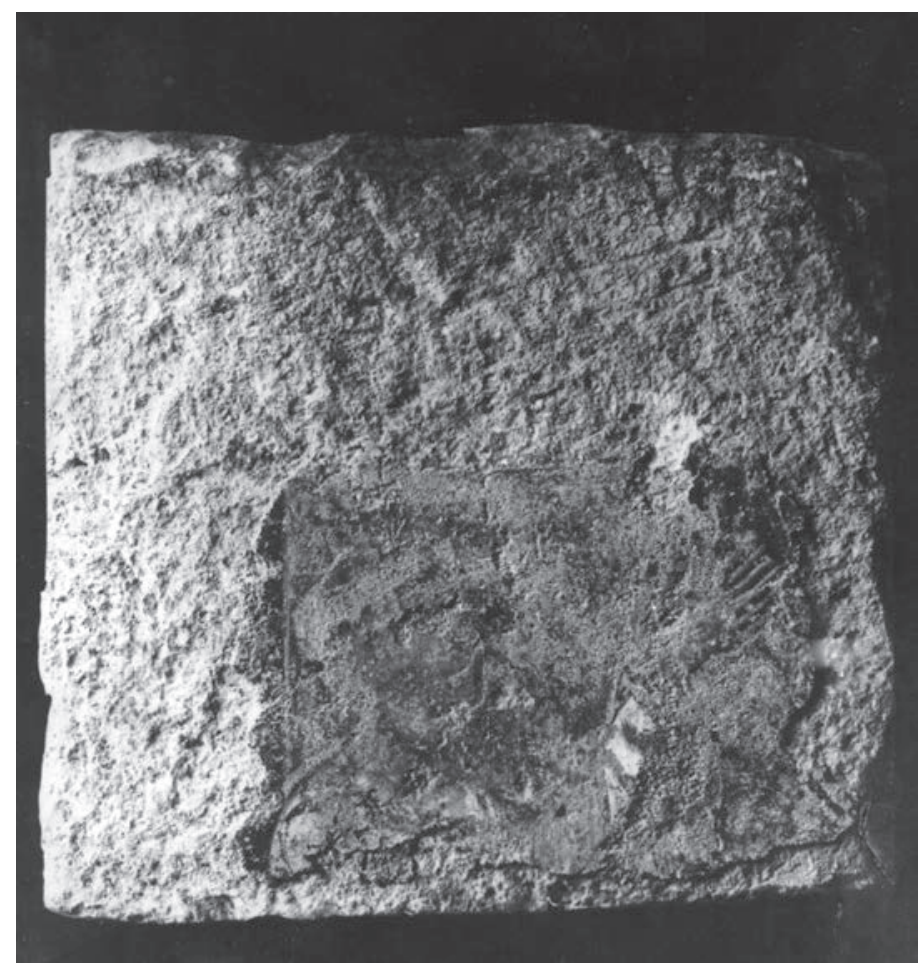

Fig. 2. Frammento di intonaco dipinto con figura di chierico (?). Dagli scavi di S. Maria d'Aurona (perduto).

in marmo col Sepolcro di Cristo d'inizio Quattrocento 3 . Nel settembre, prima dell'inizio delle demolizioni, emerse una stele romana iscritta ${ }^{4}$; nel gennaio dell'anno successivo la stampa quotidiana segnala il ritrovamento di «parecchie antichità romane e romano-cristiane», tra cui i "preziosi frammenti della chiesa di Aurona e del monumento sepolcrale di Teodosio [sic]»». Poco dopo si ritrova quella che allora apparve come la «scoperta più pregiata», un «piccolo quadretto su cui è dipinta a un quarto del vero una mezza figura che sembra un sacerdote, senza barba, con occhi grandi e spalancati, con veste a pieghe e colle mani levate in atto di preghiera ${ }^{6}$, una porzione di muratura che conservava ancora tracce di un affresco oggi perduto e documentato solo da una fotografia (fig. 2).

Latitando nella sorveglianza la Consulta del Museo Patrio, la prima ricognizione fu affidata all'erudito quanto sprovveduto abate Antonio Ceruti della Biblioteca Ambrosiana, che redasse una confusa relazione, unica testimonianza esistente delle operazioni che portarono al rinvenimento ${ }^{7}$. Ceruti riferisce che negli scavi si intercettarono i resti di una

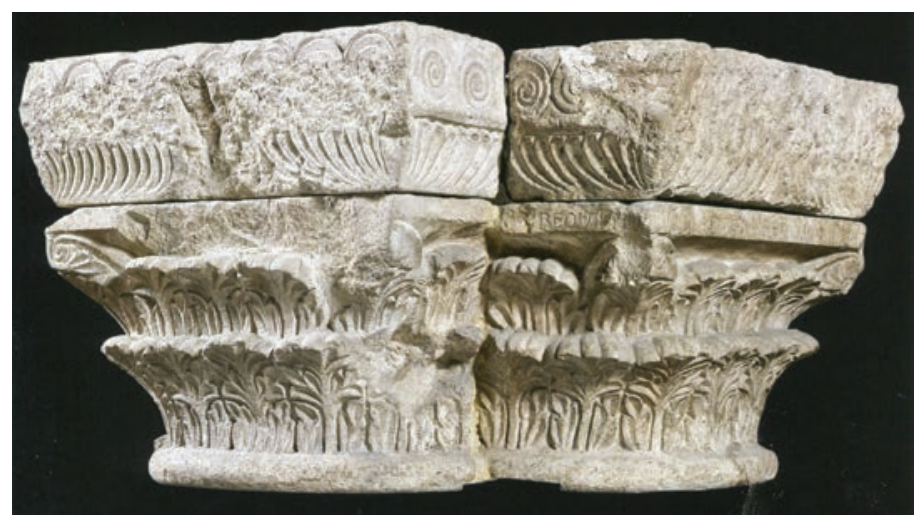

Fig. 3. Capitello della fase romanica di S. Maria d'Aurona, con iscrizione del vescovo Teodoro (Milano, Civici Musei d'Arte Antica).

torre e di un «antico e solidissimo muraglione» sviluppato per ben 30 metri, «che non poté essere distrutto se non con un lavoro assiduo di molti giorni e coll'aiuto delle mine» ${ }^{8}$. Tali tracce vennero subito collegate alla cerchia muraria tardoantica', detta "massimianea" sulla base del ritrovamento, ma in utilizzo secondario, di una base di statua con iscrizione dedicatoria ${ }^{10}$, che tutt'al più può costituire un terminus post quem, e limitatamente al tratto individuato, della sua costruzione (indicando piuttosto un intervento di restauro).

I materiali riemersi vennero riferiti al monastero di Santa Maria d'Aurona, fondato secondo la tradizione nella prima metà dell'VIII secolo e legato alla memoria del vescovo Teodoro e della sorella Aurona ${ }^{11}$. Decisiva per l'identificazione fu la lettura, sull'abaco di un capitello di pilastro polilobo (pertinente però alla fase romanica della chiesa, fig. 3), di un'iscrizione (apparentemente funeraria) menzionante un vescovo Teodoro «qui iniuste fuit damnatus $»^{12}$, identificato con l'omonimo vescovo citato nel Versus de Mediolano civitate, morto intorno alla metà dell'VIII secolo e sepolto appunto nel monastero ${ }^{13}$. I primi indagatori furono così indotti ad attribuire a quel secolo gran parte dei materiali lapidei, collegandoli alla chiesa del monastero nel quale, secondo la testimonianza di Paolo Diacono, si sarebbe rifugiata Aurona dopo la mutilazione inflittale da Aripert ${ }^{14}$.

I pezzi, «impiegati per i muri o sparsi sotterra», furono «per ordine dell'Amministrazione della Cassa di Risparmio colla massima sollecitudine raccolti dall'Ufficio Tecnico della stessa sotto la direzione del cav. Giuseppe Balzaretto $»^{15}$ e depositati temporaneamente in un magazzino. Gaetano Landriani, già collaboratore di Fernand de Dartein nella rilevazione grafica della basilica di Sant'Ambrogio, iniziò

\footnotetext{
3 Riprodotto in P. MEZZANOTTE, G.C. BASCAPE், Milano nell'arte e nella storia, Milano, 1948, p. 837.

${ }^{4}$ La Perseveranza, 4 settembre 1868.

${ }^{5}$ Ibid., 20 gennaio 1869.

${ }^{6}$ Il Pungolo, 2 marzo 1869.

${ }^{7}$ A. CERUTI, Sulle antiche mura milanesi di Massimiano, in Miscellanea di Storia Italiana, VII, Torino, 1869, pp. 785-806.

${ }^{8}$ A. CERUTI, op. cit. (n. 7), p. 787 .

9 Da ultimo, M. DAVID, Burdigala e Mediolanum nell'età di Teodosio. Fonti letterarie e fonti archeologiche a confronto, in E. Mangani, A. Pellegrino (a cura di), Гı $\alpha$ to $\phi$ í̉o $\mu \alpha$ c. Scritti in ricordo di Gaetano Messineo, Roma, 2016, p. 137-145.

${ }^{10}$ R. CASSANELLI, M. DAVID, op. cit. (n. 1), p. 15.

${ }^{\text {" }}$ Per una sintesi delle fonti v. M.T. FIORIO, Santa Maria d'Aurona, in Le chiese di Milano, Milano 1985, p. 172-173.

${ }^{12}$ E. SELETTI, Marmi scritti del Museo Archeologico. Catalogo, Milano 1901, p. 211-213, n. 300.

${ }^{13}$ J.-C. PICARD, Le souvenir des évêques. Sépultures, listes épiscopales et culte des évêques en Italie du Nord des origins au Xee siècle, Rome, 1988, p. 85-87.

${ }^{14}$ PAOLO DIACONO, Historia Langobardorum, VI 22.

${ }^{15}$ G. LANDRIANI, op. cit. (n. 1), p. 83.
} 
subito a studiarli e disegnarli in situ, prima ancora che le demolizioni fossero portate a termine ${ }^{16}$. Le sue indicazioni a favore dell'ancoraggio all'epoca "lombarda" furono subito accolte da de Dartein nel suo Étude sur l'architecture lombarde (1865-82), nel quale la struttura, ipoteticamente ricostruita, di S. Maria d'Aurona era elevata a precedente del sistema voltato del S. Ambrogio, anch'esso anticipato al IX secolo, e interpretata come una sorta di laboratorio della sua decorazione plastica ${ }^{17}$. Esclusa la possibilità che i capitelli «un tempo sostegno della tomba dell'arcivescovo di Milano Teodoro II» fossero trasferiti in Duomo ${ }^{18}$, i pezzi, donati nell'estate 1869 al Museo Patrio di Archeologia ${ }^{19}$, vennero collocati nelle navate della chiesa sconsacrata di S. Maria di Brera, allora sede del Museo, incongruamente mescolati secondo la disposizione suggerita da Landriani ${ }^{20}$. Nella guida del Museo realizzata in oc-

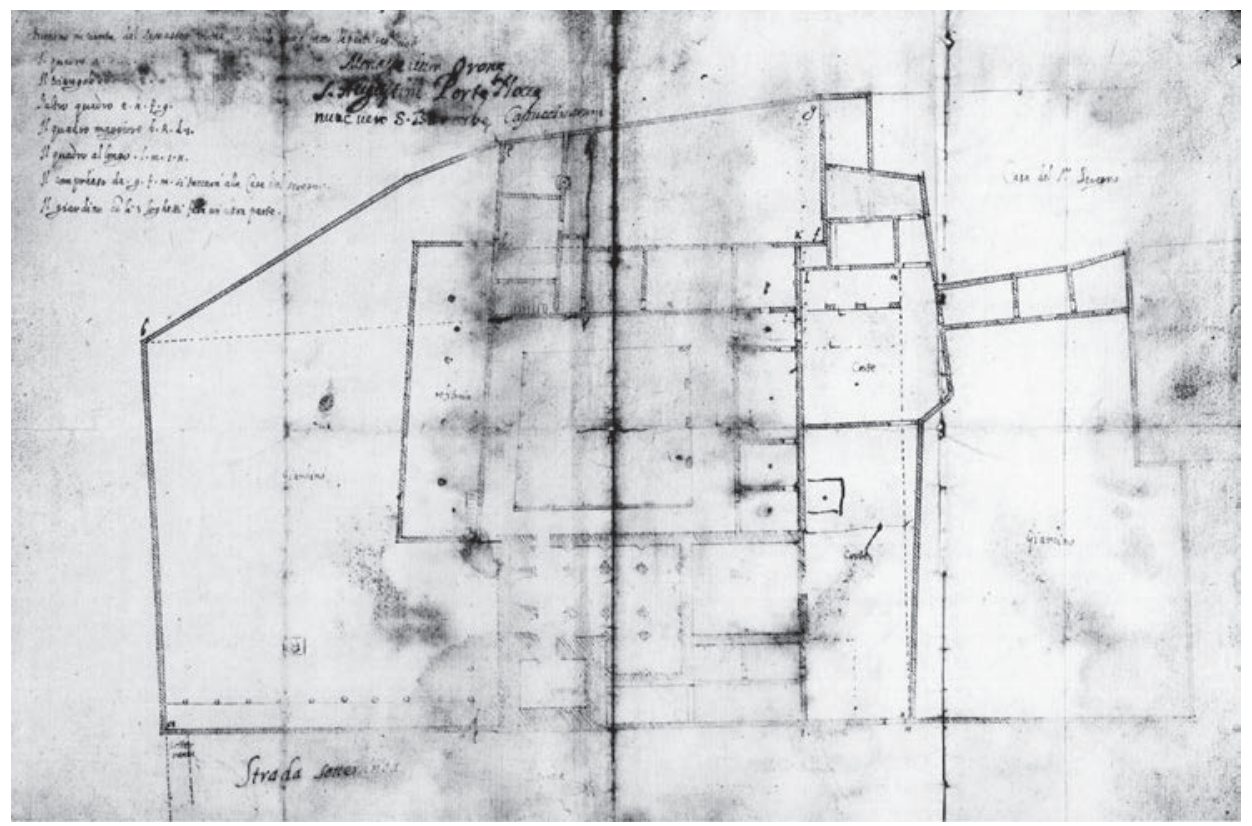

Fig. 4. Planimetria tardocinquecentesca del monastero di S. Maria d'Aurona (Milano, Archivio Storico Diocesano). casione dell'Esposizione Nazionale del 1881, si menzionano cumulativamente «duecentosessantatre pezzi, tra lapidi pagane e cristiane, capitelli di pilasti a fascio ed altri di fusto semplice, membrature architettoniche, stucchi dipinti ecc. rinvenuti negli scavi praticati in via Monte di Pietà, ove sorgeva l'antica chiesa di Santa Barbara nel monastero di Orona» ${ }^{21}$.

Così, «mescolati senz'ordine», vide i materiali Raffaele Cattaneo $(1889)^{22}$, il rinnovatore degli studi sull'architettura e la scultura dell'altomedioevo in Italia ${ }^{23}$. Sulla base di considerazioni di coerenza strutturale e stilistica, Cattaneo affermò in particolare l'esigenza di una più chiara distinzione cronologica dei pezzi - cui d'altra parte era autonomamente pervenuto anche Giuseppe Mongeri ${ }^{24}$-, che articolò in due gruppi principali, riferiti rispettivamente all'VIII-IX e all'XIXII secolo ${ }^{25}$, attirandosi la netta contrarietà di Luca Beltrami, tenace assertore di una datazione cumulativa "alta"26. A questa posizione si oppose Arthur Kingsley Porter, rifondatore degli studi sul romanico lombardo, che nell'ambito della sistematica catalogazione allargò ulteriormente la forbice cronologica, creando ben cinque partizioni scaglionate tra il 500 e il 110o, nelle quali però mancava singolarmente proprio l'VIII secolo ${ }^{27}$. Va precisato che l'analisi dei pezzi era specialmente complicata, oltre che dallo stato frammentario (talvolta di estremo relitto) in cui erano pervenuti, dall'impossibilità di definirne adeguatamente la funzione originaria nella struttura di provenienza, della quale si continuava a ignorare l'articolazione (benché Landriani fosse riuscito, pur con qualche imprecisione, a ricostruire una campata della fase romanica ${ }^{28}$.

A sciogliere l'enigma giunse nel 1944 la scoperta, nell'Archivio della Curia di Milano (attuale Archivio Storico Diocesano), da parte di Alberto de Capitani d'Arzago, di una pianta della zona della contrada dei Tre Monasteri nella seconda metà del XVI secolo (1576-1583 ca., fig. 4), redatta in funzione della parcellizzazione e vendita a privati del complesso, da

\footnotetext{
${ }^{16}$ Gli appunti e i disegni, rimasti a lungo inediti, furono pubblicati, dopo la morte di Landriani (1899), da Luca Beltrami solo nel 1902, con prefazione di Fernand de Dartein, come estremo tentativo di riproporre una datazione ormai criticamente superata, vd. R. CASSANELLI, M. DAVID, op. cit. (n. 1).

${ }^{17}$ F. DE DARTEIN, Étude sur l'architecture lombarde, Paris, 1865-82.

${ }^{18}$ R. LA GUARDIA, L’Archivio della Consulta del Museo patrio di archeologia di Milano (1862-1903), Milano, 1989, nn. 1763, 1765.

${ }_{19} \mathrm{Nel}$ catalogo manoscritto del Museo, ai nn. 1881-1918, sono registrati 17 frammenti di iscrizioni, 136 di sculture ornamentali e architettoniche e un frammento di dipinto a fresco.

${ }^{20} \mathrm{Nel} 1870$ la Cassa di Risparmio consegnò al Museo anche le 154 monete rinvenute nella medesima occasione.

${ }^{21}$ Notizie sul Museo Patrio Archeologico in Milano, Milano, 1881, p. 20, n. 66.

${ }^{22}$ R. CATTANEO, L'architettura in Italia dal secolo VI al Mille circa. Ricerche storico-critiche, Venezia, 1889, p. 118.

${ }^{23}$ Giovane architetto, aveva progettato la sepoltura di Pio IX in S. Lorenzo fuori le mura a Roma. Sulla sua personalità, oltre all'invecchiata monografia di F. GIAVARINI, L'architetto Raffaele Cattaneo, Rovigo 1941, e alla "voce" di E. BASSI, in Dizionario Biografico degli Italiani, XXII, 1979, p. 481-83, v. A. BALLARDINI, "Da ornamento a monumento. La scultura altomedievale nella storiografia di secondo Ottocento", in Medioevo immagine e memoria, atti del convegno (Parma 2008), Milano 2009, pp. 129-136.

${ }^{24}$ G. MONGERI, L'arte in Milano, Milano 1872, p. 454-457.

${ }^{25}$ R. CATTANEO op. cit. (n. 22).

${ }^{26}$ L. BELTRAMI, Raffaele Cattaneo e la sua opera «L'architettura in Italia dal secolo VI al Mille circa», in Archivio Storico dell'Arte, II, 1889, XI-XII, p. 3-12; cfr. anche L. BELTRAMI, Gli avanzi della basilica di Santa Maria in Aurona disegnati dall'arch. Gaetano Landriani e conservati nel Museo del Castello di Milano, in Rassegna d'Arte, II, 1902, 4, p. 56-59.

${ }^{27}$ Lo studioso riteneva infatti che gran parte dei materiali ritenuti coevi alla fondazione dell'edificio costituissero in realtà dei reimpieghi; A.K. PORTER, Lombard architecture, II, New Haven, 1915-17, p. 520-530.

${ }^{28}$ G. LANDRIANI, op. cit. (n. 1), p. 26.
} 


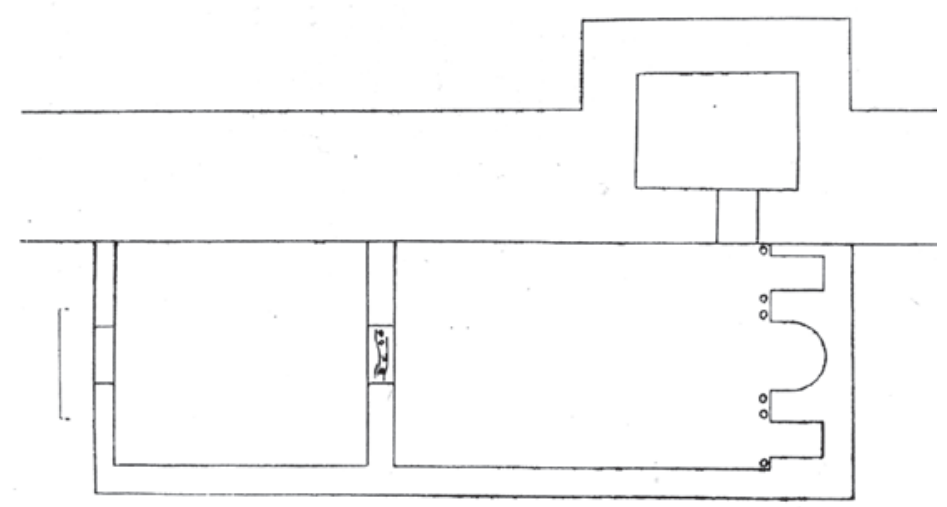

Fig. 5. Planimetria della fase longobarda di S. Maria d'Aurona (da De Capitani d'Arzago 1944). cui si poté finalmente comprendere l'articolazione del monastero e della chiesa a quell'altezza cronologica ${ }^{29}$ (un'ulteriore planimetria è stata resa nota in seguito da Luciano Patetta) ${ }^{30}$. De Capitani non ebbe difficoltà, in particolare sulla base della soluzione della parete absidale, con tre nicchie in spessore di muro, a percepire la persistenza di un edificio più antico e a proporre una ricostruzione sia della planimetria di età longobarda (fig. 5) sia della successiva rielaborazione romanica (fig. 6). Tale restituzione, che costituisce un riferimento imprescindibile, è stata sostanzialmente accettata, anche se con qualche riserva ${ }^{31}$.

Restava aperto il problema di come avessero convissuto i materiali delle due distinte fasi, considerando le probabili rielaborazioni intermedie, ad esempio dell'arredo liturgico, anche ravvicinate, che alcuni pezzi testimoniano (figg. 7-8-9).

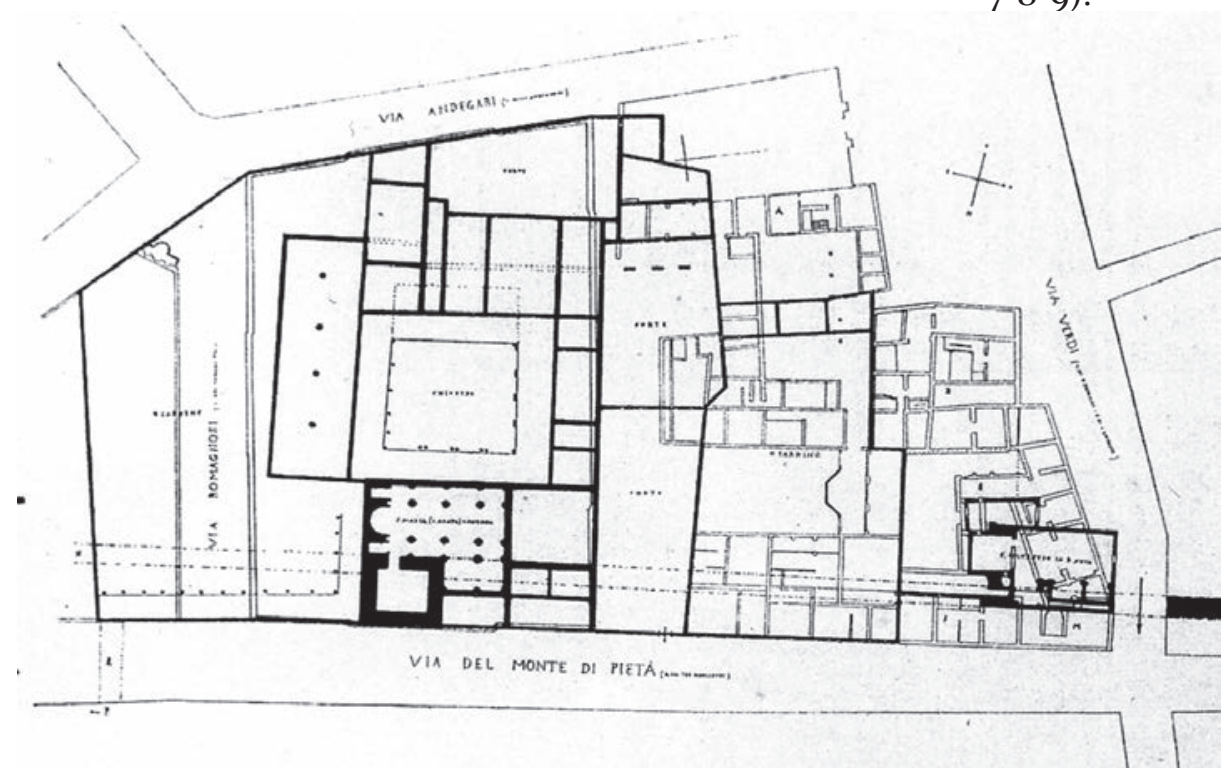

Fig. 6. Lisolato moderno nel 1930 con iscritta la pianta della chiesa di S. Maria d'Aurona nella fase romanica (da De Capitani d'Arzago 1944).
Dopo i cenni, negli anni della seconda guerra mondiale, di Rudolf Kautzsch, acuto indagatore della scultura dell'altomedioevo in Italia ${ }^{32}$, e di Paolo Verzone ${ }^{33}$, il contributo decisivo si ebbe nel 1954, quando Wart Arslan ricondusse il nucleo di sculture riferibili all'VIII secolo alla vasta categoria della "rinascenza liutprandea", nell'efficace triangolazione tra le testimonianze della scultura degli edifici religiosi delle sedi regie di Pavia e Brescia ${ }^{34}$. In tal senso se ne valse comparativamente Adriano Peroni in relazione agli stucchi del S. Salvatore ${ }^{35}$. Tralasciando le citazioni più o meno estese nelle trattazioni di più ampio respiro ${ }^{36}$, occorre giungere al 1989 per un nuovo tentativo di riconsiderazione dei materiali ${ }^{37}$, non esente da mende, cui ha fatto seguito una tesi, rimasta inedita ${ }^{3}$, nella quale si è proceduto a una puntuale revisione e catalogazione dei pezzi ${ }^{39}$, che

\footnotetext{
${ }^{29}$ A. DE CAPITANI D’ARZAGO, La chiesa romanica di Santa Maria di Aurona in Milano, da una planimetria inedita del secolo XVI, in Archivio Storico Lombardo, n.s. IX, LXXI, 1944, p. 3-66. Allo studioso spetta anche la segnalazione di due ulteriori capitelli romanici rinvenuti nel 1932 nello scavo del nuovo palazzo della Cassa di Risparmio prospiciente via Verdi, e riferiti al cenobio di Aurona, cfr. A. DE CAPITANI D’ARZAGO, Capitelli inediti di S. Maria d'Aurona, in Bollettino del Gruppo lombardo del Centro Nazionale di Studi di Storia dell'Architettura, I, 1942, 2, p. 1-18.

$3^{\circ}$ L. PATETTA, Tracce e rilevamenti di antichi edifici di Milano nei disegni del Seicento, in P. Carpeggiani e L. Patetta (a cura di), Il disegno di architettura, Atti del convegno (Milano 1988), Milano, 1989, p. 223-230.

${ }^{31}$ Cfr. in part. P. DIANZANI, Santa Maria d'Aurona a Milano. Fase altomedievale, Firenze, 1989 (Università degli Studi di Torino, Fondo di Studi PariniChirio. Studi e materiali di archeologia, 3) e F. RAVAGLIA, Santa Maria d'Aurona: una nuova lettura della scultura altomedioevale, tesi di laurea, Università Cattolica del S.C., Milano, 1997-98, rel. S. Lusuardi Siena.

${ }^{32}$ R. KAUTZSCH, Die langobardische Schmuckkunst in Oberitalien, in Römisches Jahrbuch für Kunstgeschichte, 5, 1941, p. 1-48.

33 P. VERZONE, L'arte preromanica in Liguria e i rilievi decorativi dei "secoli barbari", Torino, 1945.

${ }^{34}$ E. ARSLAN, L'architettura in Italia dal 568 al Mille, in Storia di Milano, II, Milano, 1954, pp. 499-608.

35 G. PANAZZA, A. PERONI, La chiesa di S. Salvatore in Brescia, Milano, 1962 (Atti dell'VIII congresso di studi sull'arte dell'alto medioevo); A. PERONI, I capitelli di S. Salvatore a Brescia e il problema dei capitelli preromanici di tipo corinzio, in Arte in Europa. Scritti di storia dell'arte in onore di Edoardo Arslan, I, Milano, 1966, p. 177-187; A. PERONI, Architettura e decorazione nell'età longobarda alla luce dei ritrovamenti lombardi, in La Civiltà dei Longobardi in Europa, Atti del convegno internazionale (Roma-Cividale, 1971), Roma, 1974, p. 331-359.

${ }^{36} \mathrm{Cfr}$. ad es. A.M. ROMANINI, La scultura pavese nel quadro dell'arte preromanica di Lombardia, in Pavia capitale di regno, Atti del IV Congresso internazionale di studi sull'alto medioevo (Pavia-Scaldasole-Monza-Bobbio, 1967), Spoleto, 1969, p. 231-271, ristampato in A.M. ROMANINI, Arte medievale: interpretazioni storiografiche, A. Peroni e M. Righetti (a cura di), Spoleto, 2005, p. 3-43; S. CASARTELLI NOVELLI, Nota sulla scultura, in I Longobardi e la Lombardia, Milano, 1978, p. 75-84; A. PERONI, L'arte nell'età longobarda, in Magistra Barbaritas, Milano, 1984, p. 229-297; R. CASSANELLI, Materiali lapidei a Milano in età longobarda, in C. Bertelli (a cura di), Il Millennio ambrosiano. Milano, una capitale da Ambrogio ai Carolingi, Milano, 1987, p. 238257; R. CORONEO, Scultura altomedievale in Italia, Cagliari, 2005.

${ }^{37}$ P. DIANZANI, op. cit. (n. 31).

$3^{8}$ F. RAVAGLIA, op. cit. (n. 31).

39 F. RAVAGLIA op. cit. (n. 1), p. 85-110.
} 

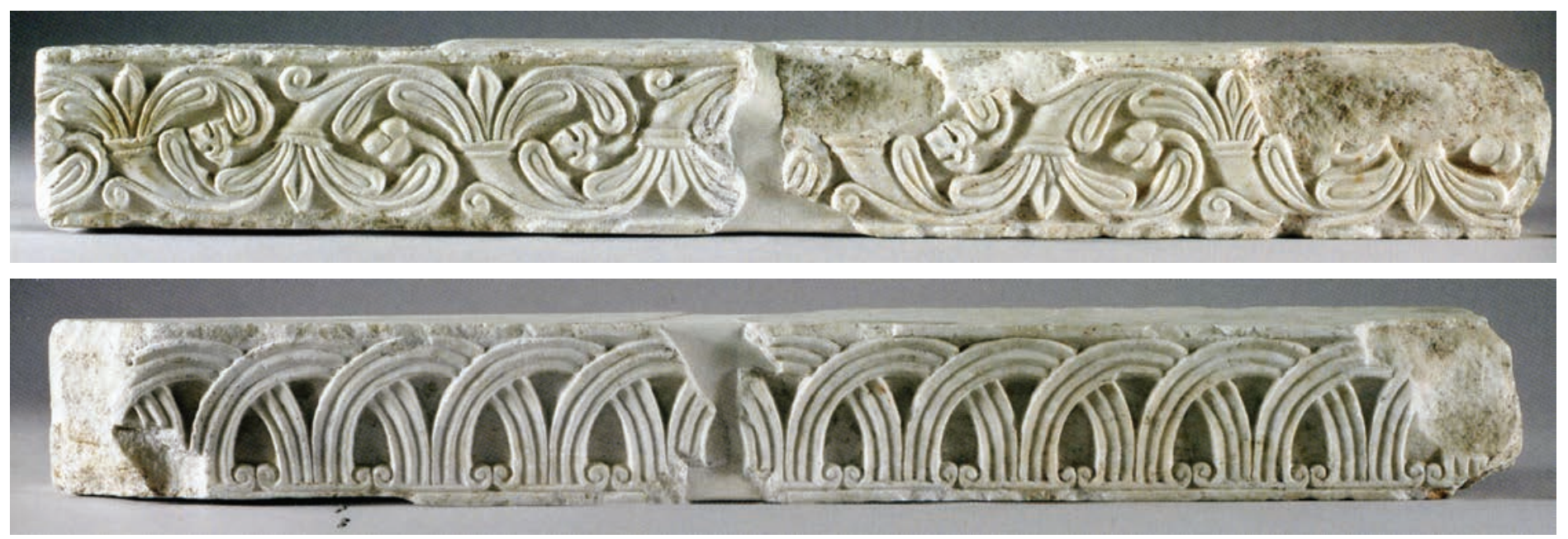

Figg. 7-8. Frammento di cornice marmorea con decorazione a tralcio vegetale di VIII sec. (faccia A) e rilavorazione successiva ad archetti intrecciati (faccia B) che ne inverte il senso di lettura, dagli scavi di S. Maria d'Aurona (Milano, Civici Musei d'Arte Antica).

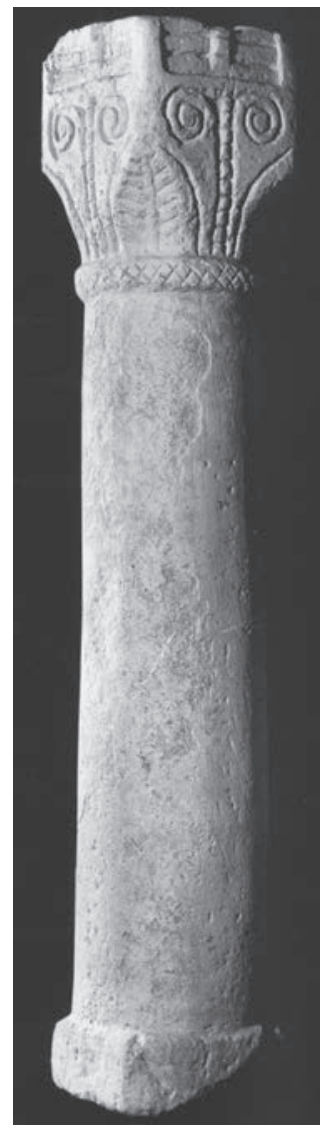

Fig. 9. Colonnina di recinzione presbiteriale, pietra, XI sec., dagli scavi di S. Maria d'Aurona (Milano, Civici Musei d'Arte Antica). intanto avevano cominciato a circolare in mostre temporanee ${ }^{40}$.

Per quanto concerne le vicende museografiche, connesse strettamente a quelle critiche, va ricordato che $\mathrm{i}$ pezzi, dopo il 1899, passarono nelle nuove sale del Museo archeologico nella Corte Ducale del Castello Sforze$\mathrm{ScO}^{41}$. In occasione di un nuovo allestimento, promosso all'inizio degli anni Trenta da Giorgio Nicodemi, vennero catalogati da Silvio Vigezzi, che ne enumerò 120, di cui 32 principali e 88 «di varie membrature architettoniche di stile lombardo [...] di varia epoca, anche del Rinascimento ${ }^{42}$. Nel riallestimento del Museo d'Arte Antica di Costantino Baroni e del gruppo BBPR (1956), tale distinzione fu assunta a criterio espositivo, selezionando drasticamente i pezzi sulla base della conservazione e leggibilità: solo pochi vennero così destinati all'esposizione, mentre il resto finì in deposito, condannati a un lungo oblio, sino almeno alla catalogazione del 2012-2014 ${ }^{43}$. Una maquette (realizzata da A. Degani sulla base dell'ipotesi ricostruttiva di A. De Capitani) integrò l'esposizione presentando la chiesa nella fase romanica ${ }^{44}$.

Il monastero femminile di S. Maria d'Aurona, di osservanza benedettina, occupava l'isolato tra le attuali vie Andegari, Monte di Pietà e Romagnosi. Le fonti tacciono riguardo la fondazione (fissata al 740 dalla Cronaca di Goffredo da Bussero, della fine del XIII secolo), presumibilmente avvenuta nei primi decenni dell'VIII secolo. Il terminus ante quem dovrebbe essere stabilito dalla morte e sepoltura nel monastero del vescovo Teodoro, sulle quali non vi è peraltro piena concordanza. La scelta del cenobio femminile per la collocazione della tomba di un vescovo è certamente singolare, e potrebbe costituire una riprova degli speciali vincoli che dovettero sussistere tra il vescovo e il personaggio il cui nome passò a designare il monastero e la sua chiesa, Aurona. Picard ha perciò ipotizzato che si sia trattato di un'inumazione di carattere privato, a causa delle oscure vicende che riguardarono il presule, e che a distanza di secoli vennero ancora richiamate nell'iscrizione apposta a un capitello collocato forse in prossimità della tomba ${ }^{45}$. La menzione, nel Versus de Mediolano civitate (739 ca.), di Teodoro come discendente da stirpe regia («natus de regali germine») ha indotto a supporre che fosse figlio di Ansprando e fratello di re Liutprando, di cui Paolo Diacono ricorda appunto una sorella di nome Aurona. L'ipotesi identificativa suggerita da tale suggestiva triangolazione è plausibile, ma ostano alcuni elementi. Paolo menziona in effetti una Aurona figlia di Ansprando che andò sposa ed ebbe due figli, ma non accenna né alla fondazione del monastero né a un suo eventuale ruolo di badessa, limitandosi a riferire della mutilazione inflittale da Aripert. Ugualmente non ricorda un Teodoro arcivescovo di Milano fratello di Liutprando (legame taciuto anche dalle liste episcopali).

La questione, che ha avuto soprattutto nella critica ottocentesca sviluppi romanzeschi, è pertanto da considerarsi ancora sub iudice. Resta il fatto della pertinenza regia del monastero, testimoniata dalla sua prima effettiva menzione

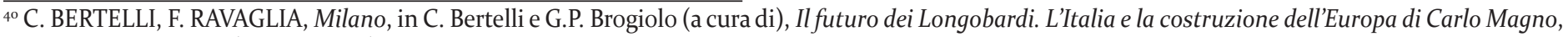
Catalogo della mostra (Brescia 200o), Milano, 200o, p. 252-259; A. BALLARDINI, Santa Maria d'Aurona, in Il medioevo delle cattedrali. Chiesa e Impero: la lotta delle immagini (secoli XI e XII), Catalogo della mostra (Parma 2006), Milano, 2006, p. 419-422.

${ }^{41}$ A. FROVA, Guida sommaria dei Civici Musei archeologico ed artistico nella Corte Ducale del Castello Sforzesco, Milano, 1906, p. 12-13.

${ }^{42}$ S. VIGEZZI, La scultura in Milano, Milano, 1934, nn. 21-52 e 53-120, p. 64.

${ }^{43}$ R. CASSANELLI, Il complesso monastico di Santa Maria d'Aurona a Milano. Fase altomedievale (VIII-IX sec.), in Museo d'Arte Antica del Castello Sforzesco. Scultura lapidea, I, Milano, 2012, p. 54-82, da integrare con M. VACCARO, Santa Maria d'Aurona. Appendice, in Museo d'Arte Antica del Castello Sforzesco. Scultura lapidea, IV, Milano, 2015, p. 132-141.

${ }_{44}^{4}$ Per iniziativa di M.T. Fiorio i frammenti in deposito vennero revisionati e fotografati alla metà degli anni Ottanta del Novecento, e ancora nel 1993 da parte dell'I.C.S.A.-Interventi Conservativi Storico-Artistici di B. Giacomelli.
}

45 J.-C. PICARD op. cit. 1988 (n. 13), p. 87. 
nel diploma dell'88o col quale Carlo il Grosso confermava la donazione a Sant'Ambrogio del «monasterium ... quod vocatur Aurune» disposta dall'imperatrice Angilperga in memoria del defunto marito Ludovico II, morto nell' $875^{46}$. Si può immaginare che sia accaduto qualcosa di analogo a quanto successo al S. Salvatore di Brescia, monastero fondato intorno al 754 da re Desiderio passato già nell' 814 sotto il controllo della famiglia imperiale. La fondazione del monastero di Aurona nella prima metà dell'VIII secolo bene si inserisce d'altra parte nella politica religiosa di Liutprando, e costituisce oltre tutto l'unica attestazione di una fondazione regia longobarda a Milano.

Nel 1081 Enrico IV riconfermò alle monache privilegi che non erano più in grado di documentare a causa del grande incendio del 1075 che aveva distrutto l'archivio e parte delle strutture monastiche ${ }^{47}$. Per quanto riguarda la chiesa, il fuoco dovette intaccare soprattutto la struttura lignea del tetto, il cui irreparabile danneggiamento indusse a mutare il sistema di coperture, optando per una serie di volte sorrette da pilastri cruciformi che tripartirono in navate l'aula sino a quel momento unica. I lavori di riorganizzazione, paralleli a quelli di altre chiese romaniche milanesi ${ }^{4}{ }^{8}$, si conclusero probabilmente intorno al 1095. Alle fine del XIII secolo il Liber notitiae menziona, nel monasterium Olone porte Nove, otto altari, dedicati ai santi Andrea, Agata, Antonio abate, Carpoforo, Michele, Pietro, Sofia e le sue tre figlie e alla Croce ${ }^{49}$. Il culto di sant'Agata dovette assumere a un certo punto un particolare sviluppo, se nel XV secolo il monastero era noto come S. Agata d'Orone.

Da questo momento ne inizia però il rapido declino. $\mathrm{Nel}$ 1472 Sisto IV soppresse l'abbazia, abitata a quel tempo da tre sole monache, cui impose il passaggio all'ordine agostiniano e la dipendenza dal convento di S. Agnese. Avendo però la badessa di S. Agnese declinato l'offerta, il papa, su richiesta del duca di Milano, unì S. Maria d'Aurona al vicino convento di S. Agostino di Vedano, da cui era separata solo dalla strada (l'attuale via Monte di Pietà), sotto la quale venne scavato un collegamento sotterraneo. Dalle planimetrie note si ricava che la chiesa aveva già subito a quell'altezza cronologica una prima trasformazione secondo lo schema tradizionale conventuale "doppio", diffuso tra Quattro e Cinquecento, con un'area riservata alle religiose e una ai fedeli, con la rielaborazione dell'atrio antistante e la collocazione di un altare al centro del setto di separazione, in corrispondenza dell'ingresso aperto nell'antica facciata, per poter seguire contemporaneamente la messa da entrambe le parti ${ }^{\circ}$. Anche questa fase ebbe però vita breve.

Circa un secolo dopo Carlo Borromeo, sciolto il rapporto con il monastero di S. Agostino di Vedano e fatta murare la strada sotterranea, acconsentì che una parte del complesso fosse venduta a tal Annibale Vistarino e alla moglie Giovanna
Anguissola per istituirvi un collegio per fanciulle povere votate alla castità (1583). Contro la volontà dei fondatori, già le prime fanciulle chiesero di essere aggregate a un ordine religioso e di prendere il velo. Sisto $\mathrm{V}$ acconsentì e istituì un nuovo convento di osservanza francescana dedicato a $S$. Barbara (1585). Superate le opposizioni dei coniugi Vistarino e delle agostiniane, che erano tornate a rivendicare l'antico possesso, il 7 novembre 1593 le cappuccine di S. Barbara fecero ingresso nel complesso, che mantennero sino alle soppressioni giuseppine del 1782 . Esse promossero il rifacimento della chiesa secondo il gusto moderno, che segnò la fine di un contesto miracolosamente conservatosi per secolis5.

È a questa occasione che va infatti ascritta la demolizione della maggior parte del complemento plastico, altomedievale e romanico, e il suo reimpiego nei muri e come riempimento nelle fondazioni della nuova chiesa. Riadattato da Giuseppe Piermarini a sede dei Luoghi pii riuniti (1784), secondo i principi di razionalità ed economicità cui era improntata la gestione amministrativa asburgica, il complesso fu destinato dai francesi e poi dagli austriaci a sede del Genio militare. Dopo l'Unità, caduto in abbandono e fortemente compromesso nelle strutture, fu ceduto alla Scuola d'incoraggiamento arti e mestieri (1859) e da questa alla Cassa di Risparmio, che decise di erigere nell'area la propria nuova sede.

La chiesa di S. Maria d'Aurona costituisce l'unica testimonianza conosciuta di architettura religiosa di età longobarda a Milano. Sulla base delle planimetrie che fissano la situazione immediatamente prima della trasformazione della fine del Cinquecento, è possibile riguadagnarne con sufficiente approssimazione l'articolazione planimetrica, anche se con dubbi non del tutto fugati. La chiesa si appoggiava alle mura del circuito tardoantico, lungo l'attuale via Monte di Pietà, inglobandone parzialmente una torre (poi trasformata in campanile e demolita intorno al 1580), il cui muro sud si allineava senza soluzione di continuità alla parete absidale rettilinea. Il piano inferiore della torre era posto in diretta comunicazione con la chiesa; si può supporre che vi fosse installata una cappella, come in altri casi coevi, oppure che fungesse da sagrestia (di cui non pare esservi traccia altrove).

Si trattava di una struttura ad aula unica (misure approssimative $16,80 \times 10,20 \mathrm{~m}$ ca.), un rettangolo allungato preceduto da un breve atrio e concluso da una parete rettilinea con tre absidi allineate - semicircolare al centro, rettangolari ai lati - ricavate in spessore di muro. La chiesa comunicava con il chiostro grosso modo trapezoidale su cui insistevano i principali edifici monastici. Si tratta di uno schema detto Dreiapsidensaal o ad aula unica triabsidata, diffuso in ambito alpino, in particolare retico ${ }^{2}$, il cui confronto più immediato è il S. Benedetto di Malles in Val Venosta, dove si replica - un cinquantennio più tardi - un analogo sistema

\footnotetext{
${ }^{46}$ G.P. PURICELLI, Ambrosiance Mediolani basilicee, ac monasterii, hodie cistertiensis, monumenta quibus historia Mediolanensis mirifice illustrata, multis ab erroribus vindicatur, Mediolani, 1645, n. 130. Rassegna delle fonti in M.T. FIORIO, op. cit. (n. 11), p. 172-173.

${ }^{47} \mathrm{~L}$. TESTI, Il monastero e la chiesa di Santa Maria d'Aurona in Milano, secoli VIII-XI-XVIII, in L'Arte, VII, 1904, p. 27-48, 104-129. Sulla fase romanica v. sotto il profilo documentario M.F. Baroni (a cura di), Le pergamene del secolo XII del Monastero di S. Maria di Aurona di Milano conservate presso l'Archivio di Stato di Milano, Milano, 1984.

${ }_{48}^{4}$ Per una rassegna cfr. R. Cassanelli, P. Piva (a cura di), Lombardia romanica. I grandi cantieri, Milano, 2010, e Lombardia romanica. Paesaggi monumentali, Milano, 2011.

${ }^{49}$ M. Magistretti e U. Monneret de Villard (a cura di), Liber notitiae sanctorum Mediolani, Milano, 1917, passim.

${ }^{50}$ A. DE CAPITANI D’ARZAGO, op. cit. (n. 29); L. PATETTA, op. cit. (n. 30$)$.

${ }^{51}$ «Più non si mirano vestigij delle antiche mura, perché si sono mutate e chiesa e monistero in moderne fabbriche con architettura da Cappuccini», C. TORRE, Il ritratto di Milano (1674), Milano, 1714, p. 271-272; cfr. anche S. LATUADA, Descrizione di Milano, V, Milano, 1737-38, p. 237-244.

${ }^{22}$ A. REINLE, Kunstgeschichte der Schweiz, I, Frauenfeld, 1968, p. 121-125; S. LOMARTIRE, Riflessioni sulla diffusione del tipo 'Dreiapsiden-saalkirche' nell'architettura lombarda dell'altomedioevo, in Hortus artium medievalium, 9, 2003, p. 417-431.
} 


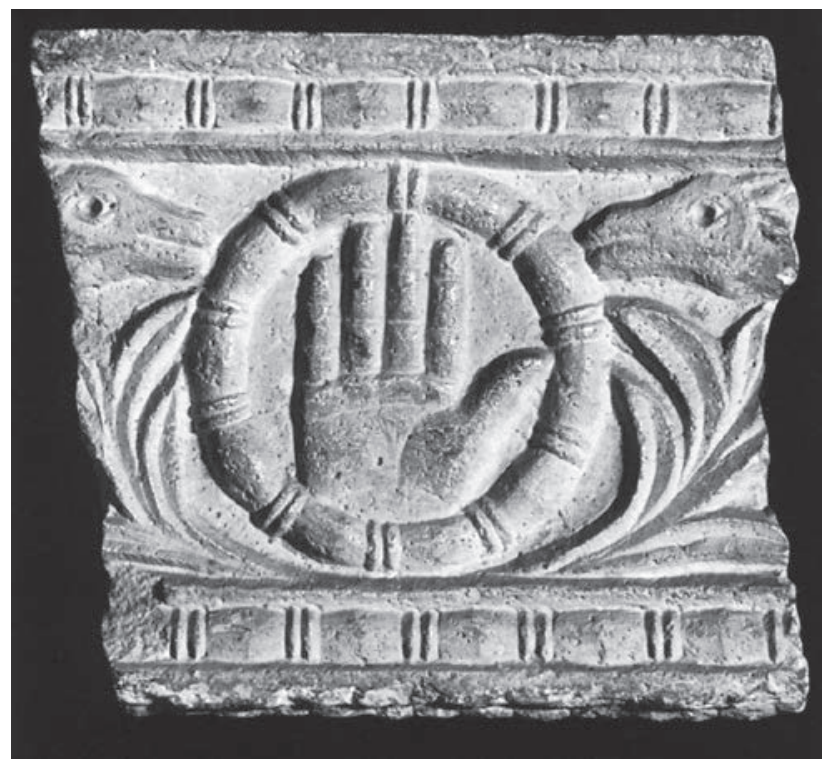

Fig. 10. Frammento di incorniciatura di porta (d'ingresso?) con "mano di Dio", pietra, metà VIII sec., dagli scavi di S. Maria d'Aurona (Milano, Civici Musei d'Arte Antica).

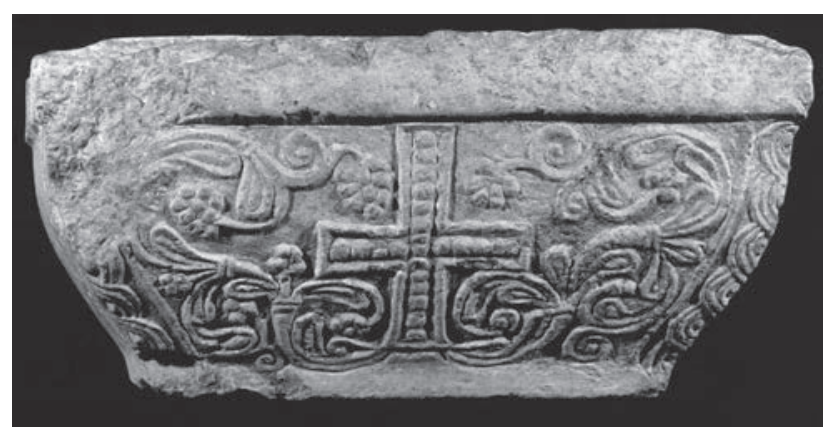

Fig. 11. Capitello-mensola, pietra, metà VIII sec., dagli scavi di S. Maria d'Aurona (Milano, Civici Musei d'Arte Antica).

di nicchie absidali con colonne "esposte", che torna anche nel St. Laurent di Grenoble ${ }^{53}$; si può richiamare a confronto anche il caso del monasterium Theodotis di Pavia (con però tre absidi estradossate) dove è riproposto il rapporto con una torre delle mura urbiche.

Irrisolto resta il problema dell'incidenza avuta sulle strutture antiche dalle trasformazioni quattro-cinquecentesche, in particolare quelle operate dalle cappuccine (sulla base delle guide sei-settecentesche parrebbe che nulla dell'antico edificio sacro fosse più conservato), e di come nella fase romanica il corredo plastico altomedievale abbia potuto convivere con quello nuovo, se sia stato cioè mantenuto (e in quale misura) in situ - come in parte parrebbe -, o reimpiegato. Ancora dall'esame delle planimetrie sembra emergere che la ridistribuzione degli spazi non interessò se non marginalmente l'originario assetto, in particolare della parete absidale, mentre per quanto concerne l'arredo liturgico vanno considerate le esigenze di adeguamento funzionale sia del periodo carolingio sia di quello romanico.

È probabile che l'incorniciatura della porta d'ingresso sia stata rimossa in occasione della trasformazione quattrocentesca dell'atrio e della chiesa in due aule distinte. Sopravvivono ancora tra i materiali pervenuti porzioni significative dell'in-

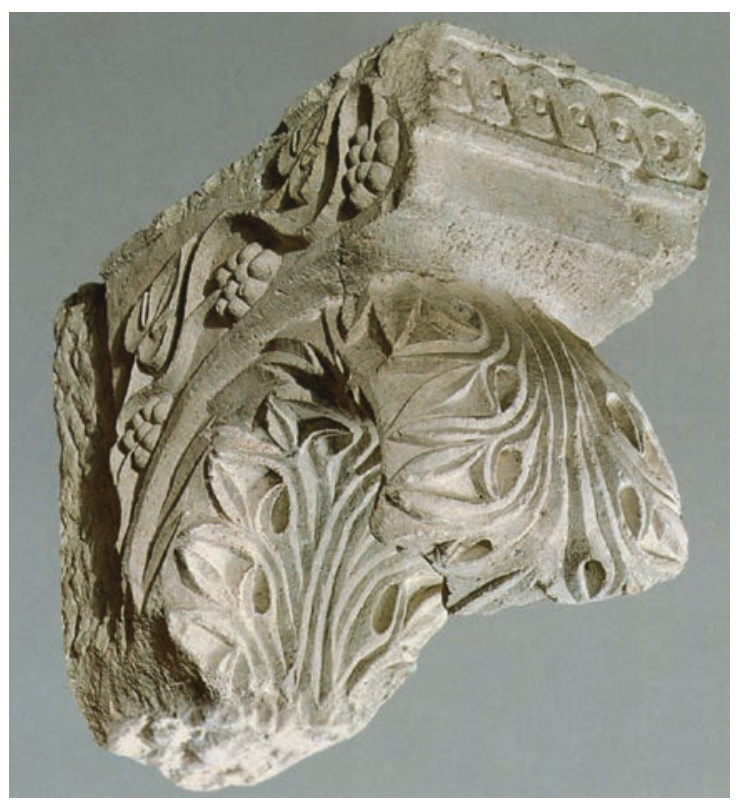

Fig. 12. Mensola, pietra, metà VIII sec., dagli scavi di S. Maria d'Aurona (Milano, Civici Musei d'Arte Antica).

corniciatura superiore e degli stipiti (fig. 10). Al centro della faccia a vista, delimitata lungo i bordi da una cornice formata da due listelli entro cui è una sequenza di ovoli eastragali, è un tondo all'interno del quale o è la "mano di Dio" aperta, con ai lati in posizione speculare due animali (probabilmente agnelli) adoranti, di cui sopravvive solo il capo ${ }^{54}$. Sulla base dell'andamento orizzontale dei motivi e della posizione della seconda faccia decorata, A causa delle ridotte dimensioni, che non ne accreditano un ruolo strutturale, il pezzo è probabilmente da riferire non tanto ad un architrave quanto all'incorniciatura superiore di un portale, probabilmente quello d'ingresso. La delimitazione a ovoli e astragali, di ascendenza classica, è particolarmente diffusa nella plastica pavese dell'VIII secolo (ad es. l'incorniciatura della specchiatura centrale delle c.d. «lastre di Teodote» dal monastero della Pusterla; Pavia Musei Civici), mentre per gli agnelli adoranti si può richiamare il confronto, anche se accentuatamente "fuori scala", con il c.d. "cavallino" di Corteolona (in realtà proprio un agnello), unico elemento superstite di un pluteo proveniente dalla cappella del palazzo di Liutprando, databile entro il $744^{55}$. Il motivo largamente diffuso della "mano di Dio" compare invece sulla fronte dell'altare di Ratchis a Cividale (733-744), e in pittura, oltre che nell'oratorio di S. Maria in Stelle presso Verona, a Pavia nella decorazione della tomba della badessa Ariperga (lato ovest) nella chiesa del monastero di S. Felice (seconda metà VIII secolo).

I capitelli-mensola (talvolta impropriamente definiti "pulvini", fig. 11), da riferire a pilastri, o forse lesene, a sezione quadrangolare, potrebbero provenire dalla parete absidale, caratterizzata da un sistema di "colonne esposte", ma rimane aperto (e dopo le distruzioni ottocentesche del tutto insolubile) il problema della fedeltà e della esatta interpretazione della trascrizione grafica. Piuttosto numerose, e in parte ben conservate, sono le mensole, che si distinguono per la presenza di foglie di vibrante plasticità, che si inarcano assecondando la curvatura (fig. 12). La foglia estroflessa in

\footnotetext{
53 A. PERONI op. cit. (n. 35), p. 357-358.

54 Sino all'ultimo allestimento si poteva rilevare la decorazione della faccia inferiore, con una croce tra motivi fogliati, vd. R. CASSANELLI, M. DAVID, op. cit. (n. 1), p. 76; P. DIANZANI, op. cit. (n. 31), p. 40, n. XXIV e tav. XII, ha per errore "sdoppiato" il pezzo, ritenendo sulla base della fotografia la faccia oggi celata un frammento autonomo perduto.

55 R. CASSANELLI, Il 'cavallino' da Corteolona nei Musei Civici di Pavia, in Bollettino della Società Pavese di Storia Patria, n.s., XC, 42, 1990, p. 289-294.
} 


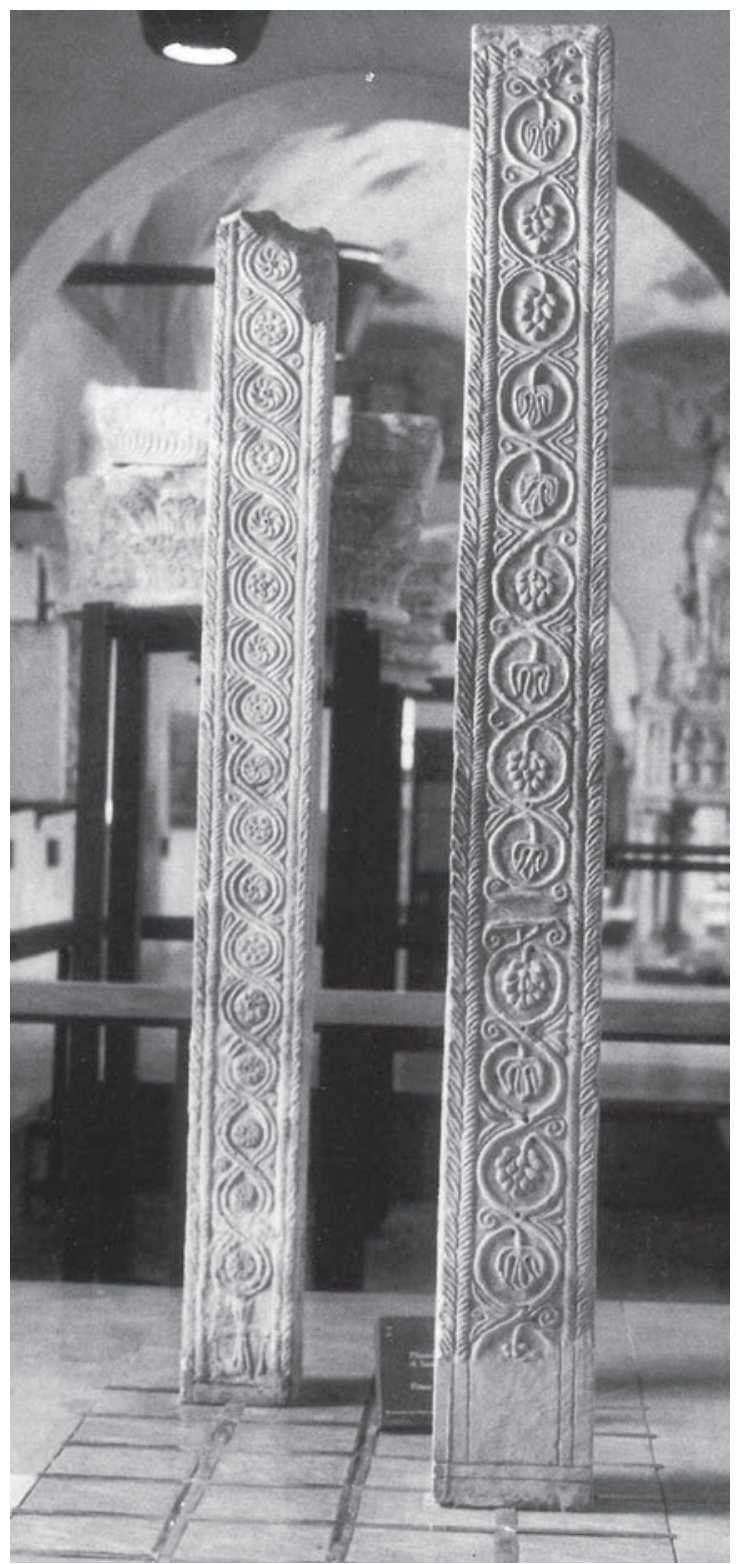

Fig. 13. Pilastrini di recinzione presbiteriale (?), pietra, metà VIII sec., dagli scavi di S. Maria d'Aurona (Milano, Civici Musei d'Arte Antica).

aggetto costituisce un unicum nella plastica altomedievale dell'Italia settentrionale, trovando un possibile confronto tipologico solo nella Langobardia meridionale, a Montecassino, e a Cimitile (Nola) nel protiro della basilica dei Ss. Martiri (datato su base epigrafica al 900 ca.). Non è possibile allo stato attuale proporre una possibile collocazione nell'edificio della serie di mensole, né ipotizzarne la funzione; se reggessero cioè una trabeazione o si collegassero a un tratto di particolare rilievo della travatura lignea del tetto, ad esempio in prossimità della parete absidale.

Dell'arredo liturgico sopravvivono due alti pilastrini, lievemente rastremati, ricomposti per l'intera altezza e decorati sulle quattro facce (fig. 13), che non trovano immediati confronti nella Langobardia maior. Il riferimento più persuasivo è costituito, oltre che dai sostegni del protiro

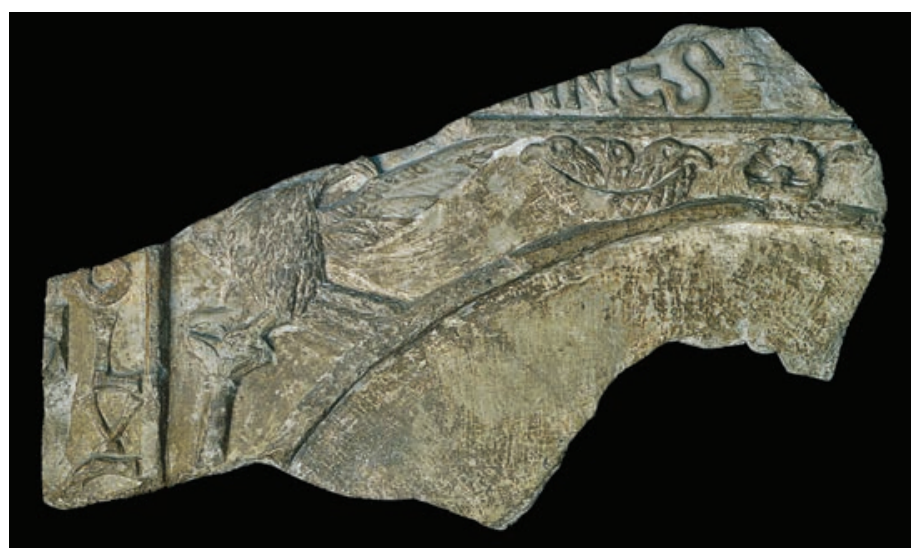

Fig. 14. Frammento di lastra iscritta, pietra, metà VIII sec., dagli scavi di S. Maria d'Aurona (Milano, Civici Musei d'Arte Antica).

della basilica dei Ss. Martiri a Cimitile, già evocato a proposito della prima serie di mensole fogliate, dalla recinzione presbiteriale dell'oratorio di S. Aspreno a Napoli, nel quale il valico di accesso al presbiterio è appunto segnato da pilastrini rastremati a sezione quadrangolare ornati da tralci. I motivi attestati sono particolarmente diffusi nella plastica di VIII secolo tra Brescia e Pavia: il tralcio di foglie baccellate nascenti da un vaso si ritrova ad esempio in un pilastrino da S. Pietro in Ciel d'Oro, mentre reimpiegata nel monastero di S. Salvatore di Brescia è una colonnetta interamente rivestita da un tralcio con foglie di vite e grappoli alternati (Panazza, Tagliaferri 1966, pp. 83-84 n. 91).

Di particolare interesse sono gli esigui frammenti di quella che potrebbe interpretarsi come una fronte di altare o di pluteo (fig. 14). Della lastra, di forma probabilmente rettangolare, sopravvive la sola porzione superiore sinistra. Una larga cornice, segnata da un doppio listello aggettante, delimitava il campo centrale e conteneva iscrizioni a rilievo. Nella fascia laterale sinistra si leggono, disposte verticalmente, le lettere] O (?) LVC[; in quella superiore: ]NNES + S (?). Il campo centrale, delimitato da un arco di cerchio formato da un listello rilevato (una mandorla?), è quasi completamente perduto. Sopravvive lo spazio di risulta superiore di sinistra, occupato da un'aquila ad ali spiegate col capo rivolto a destra che poggia su un'alta, sottile colonna inclinata, e da un nido con tre aquilotti ai lati di una conchiglia (che segna probabilmente il punto mediano della composizione), oltre la quale è il capo di un altro aquilotto (e quindi probabilmente vi si trovava un altro nido simmetrico al precedente). Gianpiero Bognetti ha proposto di integrare le lettere in lacuna delle iscrizioni (AGIO]S LVC[AS e (AGIOS) IOHA]NNES, datate sulla base di osservazioni paleografiche alla fine del VII-inizi dell'VIII secolo ${ }^{56}$.

Se da un lato Bognetti ha richiamato insistentemente $\mathrm{i}$ nessi del frammento con la cultura siriaca (cui riconduceva a suo avviso anche l'impianto della chiesa), dall'altro ne ha evidenziato le somiglianze, per il netto stacco dal fondo e la forte ascendenza classicista, nonostante la differenza dei materiali impiegati, con la decorazione del c.d. dittico di Davide e Gregorio nel Tesoro del Duomo di Monza, avorio

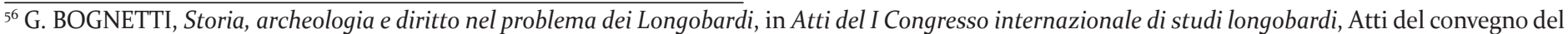
Centro italiano di studi sull'alto medioevo (Spoleto 1951), Spoleto, 1952, p. 71-136, poi in ID., L'età longobarda, III, Milano, 1967, p. 197-266. P. DIANZANI, op. cit. (n. 31), p. 55, seguendo i suggerimenti di G. Fissore, ha messo in dubbio le argomentazioni paleografiche e il conseguente ancoraggio cronologico di Bognetti, ipotizzando una possibile loro estensione «fino all'età romanica. F. RAVAGLIA, op. cit. (n. 31), p. 181 ha invece ipotizzato per la scritta verticale l'integrazione [(S.) PA]OLV(S) e per quella orizzontale [(S.) IOHA]NNES.
} 


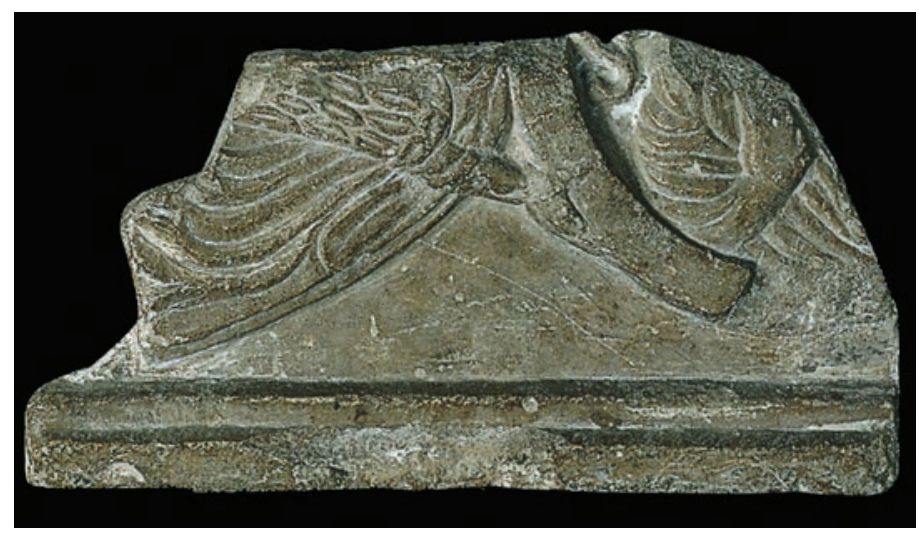

Fig. 15. Frammento di lastra, pietra, metà VIII sec., dagli scavi di S. Maria d'Aurona (Milano, Civici Musei d'Arte Antica).

tardoantico rilavorato nell'VIII-IX secolo e trasformato in legatura di codice. Se in questo caso (anche per l'esiguità delle lettere sopravvissute) la paleografia non soccorre del tutto, i caratteri formali collegano il pezzo alla forte ripresa classicista tra l'età di Liuprando e Desiderio e la conquista franca, di cui sono testimonianza eloquente i rilievi del S. Salvatore a Brescia (in particolare le celebri lastre di ambone con i pavoni). Resta da interrogarsi sulla possibile funzione della lastra - per la quale è stata ipotizzata, sulla base di confronti cividalesi, la provenienza da un altare ${ }^{57}-$, forse pluteo di recinzione presbiteriale, anche in relazione alla probabile iconografia complessiva, collegata agli evangelisti, come nel pluteo di Sigvaldo, innestato nella base del battistero di Callisto a Cividale, in cui i rispettivi simboli sono inseriti in tondi annodati «a otto» ai lati della croce e dell'«albero della vita» ${ }^{8}$.
Un ulteriore frammento di minori dimensioni (fig. 15) è attualmente innestato nel medesimo supporto del precedente, ma la sua pertinenza non è peraltro affatto provata. Sopravvive solo una piccola porzione dell'angolo inferiore sinistro con la figura fortemente lacunosa di un angelo in volo (di cui rimane parte della veste e dell'ala e una mano) che regge un clipeo con all'interno un personaggio panneggiato in posizione frontale benedicente. A fianco di questo, all'altezza della spalla, è in rilievo la lettera « $\mathrm{S}$ » (sanctus), analoga per ductus a quella dell'iscrizione della lastra precedente. Per quanto riguarda il tema dell'imago clipeata sono stati evidenziati i legami con l'impianto della faccia principale dell'altare di Ratchis a Cividale (entro il 744) e con i rilievi di Santa Maria a Quintanilla de las Viñas (Burgos), peraltro di datazione discussa.

Altri minori frammenti possono essere ricondotti a elementi di recinzione presbiteriale (cornici superiori) e forse di un ciborio, ma le loro ridotte dimensioni impediscono di formule ipotesi che potrebbero rivelarsi azzardate.

A differenza del S. Salvatore di Brescia non sono emerse testimonianze di stucchi decorativi, materiale facilmente deperibile, mentre l'unico lacerto di pittura, molto rovinata, con la figura di un diacono (di cui Landriani tracciò il contorno e di cui rimane una fotografia), di incerta datazione (età carolingia?), è andato disperso. La qualità di alcuni pezzi, realizzati con tutta probabilità da una bottega locale sulla base di un repertorio di modelli comuni largamente circolante, ne fa uno dei punti di riferimento della c.d. "rinascenza liutprandea", in diretta relazione con la produzione delle officine attive per la corte regia a Pavia (in un arco di attività che da Corteolona si spinge sino a Bobbio) e soprattutto a Brescia, nel cantiere desideriano del S. Salvatore. 